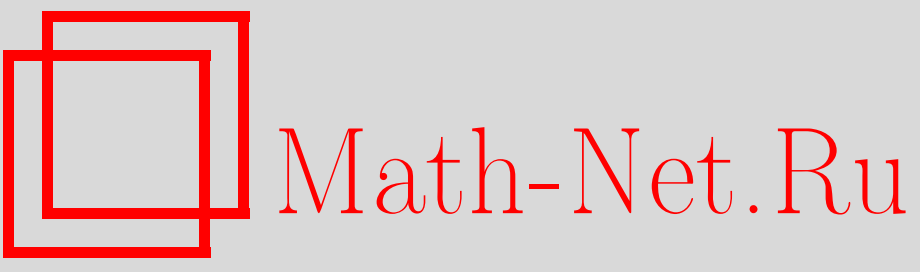

А. В. Тищенко, Сплетение атомов решетки полугрупповых многообразий, УМН, 1998, том 53, выпуск 4, 219-220

DOI: https://doi.org/10.4213/rm64

Использование Общероссийского математического портала Math-Net.Ru подразумевает, что вы прочитали и согласны с пользовательским соглашением

http://www.mathnet.ru/rus/agreement

Параметры загрузки:

IP: 54.164 .48 .24

26 апреля 2023 г., 12:05:00 


\title{
СПЛЕТЕНИЕ АТОМОВ РЕШЕТКИ ПОЛУГРУППОВЫХ МНОГООБРАЗИЙ
}

\author{
А. В. ТищЕнко
}

Операция моноидного сплетения полугрупповых многообразий была определена в [1], [2]. Моноидное сплетение полугрупповых многообразий всегда содержит их решеточное объединение (см. [3]), но может в некоторых случаях довольно сильно от него отличаться. Для получения информации о том, насколько моноидное сплетение может быть больше чем решеточное объединение этих же многообразий, показьвает исследование поведения этой операции в случае сплетения двух многообразий, каждое из которых является атомом решетки $\mathscr{L}$ полугрупповых многообразий. Отметим, что моноид полугрупповых многообразий с операцией моноидного сплетения изучался в [4].

Атомы решетки $\mathscr{L}$ хорошо известны [5]. Это в точности многообразия $\mathbf{N}_{2}$ всех полугрупп с нулевым умножением, $\mathbf{S I}$ всех полурешеток, $\mathbf{L}_{1}$ всех полугрупп левых нулей, $\mathbf{R}_{1}$ всех полугрупп правых нулей, $\mathbf{A}_{p}$ всех абелевых групп простой экспоненты $p$. Предварительно для сплетения атомов $\mathbf{U}$ и $\mathbf{V}$ решетки $\mathscr{L}$ вычисляется базис тождеств и, если это возможно, находится конечная полугруппа, которая порождает это сплетение. При вычислении базисов тождеств существенно используются результаты раздела 4 из [3]. Далее для всевозможных атомов $\mathbf{U}$ и $\mathbf{V}$ вычисляются решетки $\mathscr{L}(\mathbf{U w} \mathbf{V})$ всех подмногообразий сплетения $\mathbf{U w} \mathbf{V}$, а затем решеточные интервалы $[\mathbf{U} \vee$ $\mathbf{V}, \mathbf{U w} \mathbf{V}]$. Решеточные интервалы $[\mathbf{U} \vee \mathbf{V}, \mathbf{U w} \mathbf{V}]$ обозначим кратко через $\mathscr{T}(\mathbf{U}, \mathbf{V})$.

TeOpema 1.

1) Если $\mathbf{U}$ - идемпотент моноида $M V$ полугрупповых многообразий, то $\mathbf{U w} \mathbf{v}=$ $\mathbf{U} \vee \mathbf{V}$. В частности, $\mathbf{N}_{2} \mathbf{w} \mathbf{V}=\mathbf{N}_{2} \vee \mathbf{V} u \mathbf{L}_{1} \mathbf{w} \mathbf{V}=\mathbf{L}_{1} \vee \mathbf{V}$ для любого атома $\mathbf{V}$ решетки $\mathscr{L}$.

2) $\mathbf{R}_{1} \mathbf{w} \mathbf{N}_{2}=\operatorname{var}\left\{y_{1} y_{2} x=z_{1} z_{2} x\right\}, \mathbf{R}_{1} \mathbf{w} \mathbf{L}_{1}=\operatorname{var}\{x y z=x z\}$,

$\mathbf{R}_{1}^{2}=\operatorname{var}\left\{x y_{1} y_{2}=y_{1} y_{2}\right\}, \mathbf{R}_{1} \mathbf{w} \mathbf{S I}=\operatorname{var}\left\{x z=x^{2} z, x y z=y x z\right\}$,

$\mathbf{R}_{1} \mathbf{w} \mathbf{A}_{p}=\mathbf{R}_{1} \vee \mathbf{A}_{p}=\operatorname{var}\left\{x y z=y x z, x=y^{p} x\right\}$.

3) $\mathbf{S I w N} \mathbf{N}_{2}=\operatorname{var}\left\{x_{1} x_{2} y=x_{1} x_{2} y^{2}, x_{1} x_{2} y z=x_{1} x_{2} z y\right\}$,

$\mathbf{S I w} \mathbf{L}_{1}=\operatorname{var}\left\{x y=x y^{2}, x y z=x z y\right\}$,

$\mathbf{S I w} \mathbf{R}_{1}=\operatorname{var}\left\{x^{2}=x^{3}, x y x=x y x y x, x y x z x=x z x y x\right\}$,

SIwSI $=\operatorname{var}\left\{x^{2}=x^{3}, x y x^{2}=x y x, x y x y=x y^{2} x, x u y v x y=x u y v y x\right.$,

xuyxy $=$ xuyyx, xyvxy $=$ xyvy $\}$.

4) $\mathbf{A}_{p} \mathbf{w} \mathbf{N}_{2}=\operatorname{var}\left\{x_{1} x_{2} y z=x_{1} x_{2} z y, x_{1} x_{2} y^{p}=x_{1} x_{2}\right\}$,

$\mathbf{A}_{p} \mathbf{w} \mathbf{L}_{1}=\mathbf{A}_{p} \vee \mathbf{L}_{1}=\operatorname{var}\left\{x y z=x z y, x y^{p}=x\right\}$

$\mathbf{A}_{p} \mathbf{w} \mathbf{R}_{1}=\operatorname{var}\left\{x=x(y x)^{p}, x^{2} y x=x y x^{2},\left(x^{p} y^{p}\right)^{p}=(x y)^{p}\right\}$,

$\mathbf{A}_{p} \mathbf{w} \mathbf{S I}=\operatorname{var}\left\{x=x^{1+p}, x y v x y=x y v y x\right\}$.

5) Многообразия $\mathbf{A}_{p}^{2}$ и $\mathbf{A}_{p} \mathbf{w} \mathbf{A}_{\mathbf{q}}$, где $p$ и q- простые числа, конечно базируемы. Многообразия $\mathbf{S I w} \mathbf{A}_{p}$ ( $p$ - простое число) существенно бесконечно базируемы.

ЗАмЕчАниЕ. Конечная базируемость сплетения групповых многообразий следует из результатов известной книги [6]. Существенная бесконечная базируемость многообразий $\mathbf{S I w} \mathbf{A}_{p}$ доказана в [7]. Базис тождеств и конечная полугруппа, порождающая многообразие $\mathbf{S I w R}_{1}$, найдены в [3]. Базисы тождеств для полупрямых произведений псевдомногообразий $\mathbf{R}_{1} * \mathbf{R}_{1}, \mathbf{S I} * \mathbf{R}_{1}$ и $\mathbf{S I} * \mathbf{S I}$ были известны в литературе [8], [9].

СледСтвиЕ 1. Моноидное сплетение $\mathbf{U w V}$ атомов решетки L всех полугрупповьх многообразий не имеет конечного базиса тождеств тогда и только тогда, когда $\mathbf{U}=$ SI $u \mathbf{V}=\mathbf{A}_{p}$ для некоторого простого числа $p$.

Теорема 2. Моноидное сплетение UwV атомов решетки L всех полугрупповых многообразий не порождается конечной полугруппой тогда и только тогда, когда $\mathbf{U}=$ $\mathbf{V}=\mathbf{A}_{p}$ для некоторого простого числа $p$. 
TeOpema 3.

1) всли $\mathbf{U}=\mathbf{N}_{2}$, или $\mathbf{U}=\mathbf{L}_{1}$, или $\mathbf{U}=\mathbf{R}_{1}, u \mathbf{V}=\mathbf{A}_{p}, u л и \mathbf{U}=\mathbf{A}_{p} u \mathbf{V}=\mathbf{L}_{1}, m o$ $|\mathscr{L}(\mathbf{U}, \mathbf{V})|=4$ u $|\mathscr{T}(\mathbf{U}, \mathbf{V})|=1$.

2) $|\mathscr{T}(\mathbf{U}, \mathbf{V})|=2 u|\mathscr{L}(\mathbf{U}, \mathbf{V})|=5$.

3) $\left|\mathscr{T}\left(\mathbf{R}_{1}, \mathbf{N}_{2}\right)\right|=\left|\mathscr{T}\left(\mathbf{R}_{1}, \mathbf{R}_{1}\right)\right|=\left|\mathscr{T}\left(\mathbf{R}_{1}, \mathbf{S I}\right)\right|=\left|\mathscr{T}\left(\mathbf{S I}, \mathbf{L}_{1}\right)\right|=\left|\mathscr{T}\left(\mathbf{A}_{p}, \mathbf{N}_{2}\right)\right|$ $=\left|\mathscr{T}\left(\mathbf{A}_{p}, \mathbf{R}_{1}\right)\right|=3$. $\Pi$ пи этом $\left|\mathscr{L}\left(\mathbf{R}_{1}, \mathbf{N}_{2}\right)\right|=10,\left|\mathscr{L}\left(\mathbf{R}_{1}, \mathbf{R}_{1}\right)\right|=5,\left|\mathscr{L}\left(\mathbf{R}_{1}, \mathbf{S I}\right)\right|=\left|\mathscr{L}\left(\mathbf{S I}, \mathbf{L}_{1}\right)\right|=12$, $\left|\mathscr{L}\left(\mathbf{A}_{p}, \mathbf{N}_{2}\right)\right|=\left|\mathscr{L}\left(\mathbf{A}_{p}, \mathbf{R}_{1}\right)\right|=9$.

4) $\left|\mathscr{T}\left(\mathbf{S I w N}_{2}\right)\right|=\left|\mathscr{T}\left(\mathbf{A}_{p}, \mathbf{S I}\right)\right|=4$. При этом $\left|\mathscr{L}\left(\mathbf{S I w} \mathbf{N}_{2}\right)\right|=13,\left|\mathscr{L}\left(\mathbf{A}_{p}, \mathbf{S I}\right)\right|=11$.

5) Решетка $\mathscr{L}\left(\mathbf{A}_{p}, \mathbf{A}_{\mathbf{q}}\right)$ конечна для любых различных простых чисел $p$ и $q$.

6) Решеточные интерваль $\mathscr{T}\left(\mathbf{S I}, \mathbf{R}_{1}\right), \mathscr{T}(\mathbf{S I}, \mathbf{S I}), \mathscr{T}\left(\mathbf{S I}, \mathbf{A}_{p}\right), \mathscr{T}\left(\mathbf{A}_{p}, \mathbf{A}_{p}\right)(p-$ простое число) бесконечны.

Следствие 1 и теорема 2 были анонсированы в [10]. Теорема 3 существенно уточняет теорему 3 из [10].

\section{СПИСОК ЛИТЕРАТУРЫ}

[1] Tilson B. // J. Pure Appl. Algebra. 1987. V. 48. № 1-2. Р. 83-198. [2] Тищенко А. В. // Междунар. конф. по алгебре памяти А. И. Ширшова (Барнаул, август 1991). Тезисы докл. по логике и универс. алгебрам, прикл. алгебре. Новосибирск, 1991. С. 144. [3] Тищенко А. В. // Фундам. и прикл. матем. 1996. Т. 2. №1. С. 233-249. [4] Тищенко А. В. // УМН. 1996. Т. 51. № 2. С. 177-178. [5] Шеврин Л. Н., Волков М. В. // Изв. вузов. Матем. 1985. № 11. С. 3-47. [6] Нейман Х. Многообразия групп. М.: Мир, 1969. [7] Тищенко А. В. / Труды ММО. 1996. T. 57. C. 218-238. [8] Almeida J. // J. Pure Appl. Algebra. 1989. V. 60. P. 113-128. [9] Pin J.-E. // Semigroup Forum. V. 28. № 1-3. P. 73-81. [10] Tishchenko A. V. // Международная конф. "Полугруппы и их приложения, включая полугрупповые кольца" в честь Е. С. Ляпина. Тезисы докладов. С.-Петербург, 1995.

Принято редколлегией 03.06.1998 